\title{
Determining Lattice Parameters by Curve-Fitting Transmission Kikuchi Diffraction Patterns
}

Yueyun Chen ${ }^{1}$, Jared Lodico ${ }^{1}$, B. C. Regan ${ }^{1}$ and Matthew Mecklenburg ${ }^{2}$

${ }^{1}$ Department of Physics and Astronomy, University of California, Los Angeles, California 90095, United States, United States, ${ }^{2}$ The Aerospace Corporation, United States

Because it captures large scattering angles, Transmission Kikuchi Diffraction (TKD) is able to map crystal orientations in real time [1]. However, the algorithms currently used to determine orientation and lattice plane angles do not determine lattice parameters. Here, we introduce a new method that, by curve fitting Houghtransformed Kikuchi lines, extracts lattice plane spacings. Each pair of parallel Kikuchi lines is split by twice the Bragg angle and therefore provides the inverse lattice-plane spacing. By precisely measuring the separation of many Kikuchi lines, we generate a histogram of different lattice plane spacings. This histogram is analogous to the rotationally averaged select-area diffraction pattern produced by a polycrystalline sample in a transmission electron microscope (TEM).

Using an Oxford Symmetry EBSD camera and binning at a high gain setting, we acquire a map of $117 \times 156$ patterns of a thin, exfoliated molybdenum disulfide $\left(\mathrm{MoS}_{2}\right)$ flake (Fig. 1a) in a scanning electron microscope (SEM). Each TKD pattern contains $622 \times 512$ pixels. This acquisition creates a 4 -dimensional data set similar to a 4D scanning TEM (STEM) image. To extract precise lattice constants, we perform a Hough transform on each TKD pattern by parameterizing every line into a distance $(\rho)$ and an angle $(\theta)$ from a chosen origin. Every pixel in Hough space by definition corresponds to a line in the TKD image, so each Kikuchi band is represented as a dark-bright-dark pattern in the transformed image (Fig. 1b). In other words, bright (dark) pixels in Hough space are bright (dark) lines in the TKD image. Post-processing techniques, such as bias-correction and convolution with a butterfly-shaped kernel [2], improve the signal-to-noise ratio of the Kikuchi lines in the Hough space image enough to make curve fitting possible. We fit the dark-bright-dark patterns in Hough space with a cosine-shaped empirical model and determine the widths of the Kikuchi lines to a precision of better than $0.5 \%$ (Fig. 1c).

By repeating the processing steps described above on the entire 4D data set, we generate a real-space map of different lattice plane spacings (Fig. 1d) and a diffraction spectrum (Fig. 2). The gross shape of the sample and even-more-detailed structures can be seen clearly in the map. We achieve an agreement of $\sim 10 \%$ when comparing the measured TKD spectrums to the lattice plane spacings of crystalline $\mathrm{MoS}_{2}$ [3] (Fig. 2). The discrepancy is mainly caused by the butterfly filter, and we are working on correcting this systematic effect. This new method for extracting lattice parameters from SEM TKD 4D datasets shows great promise for characterizing electron transparent samples without the need of expensive and difficult-to-use 4D STEM techniques. [4] 

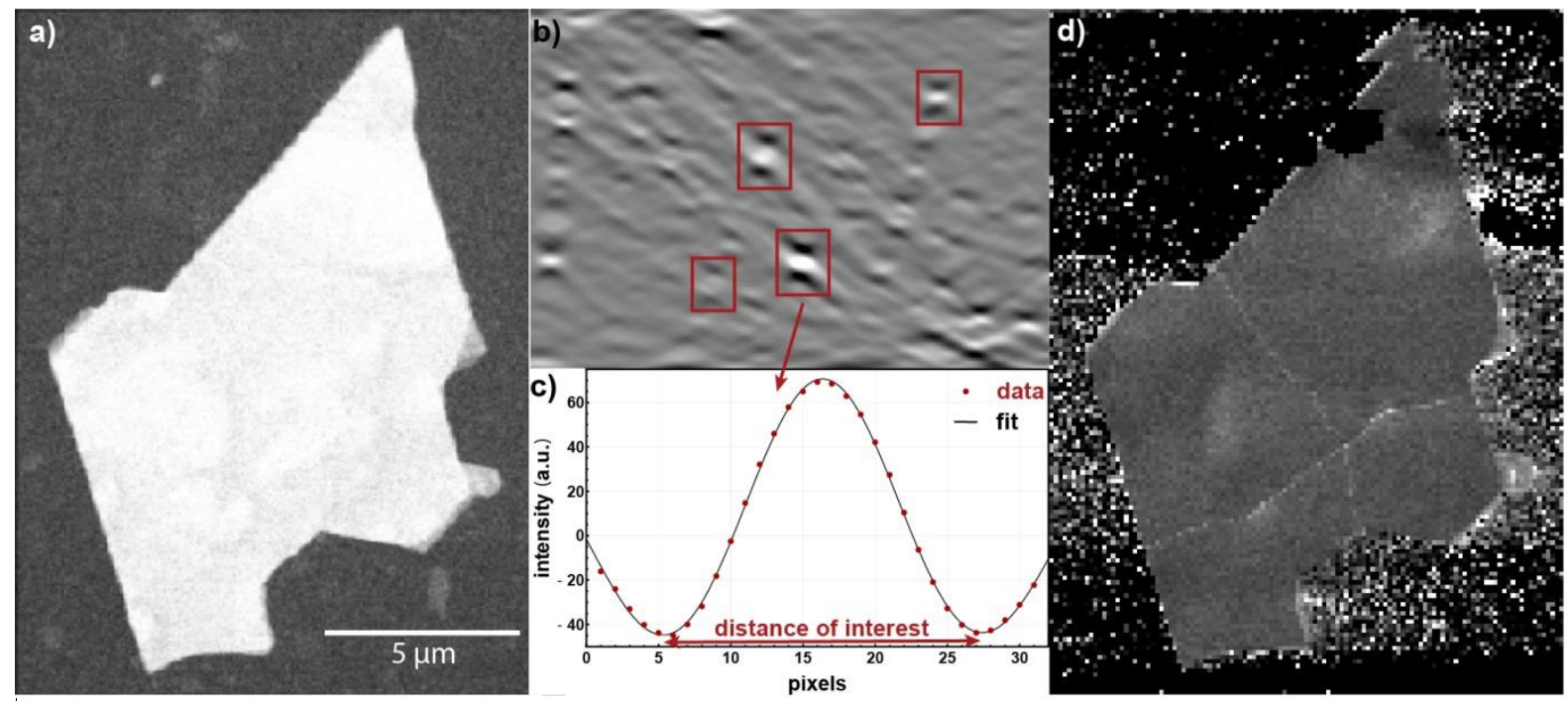

Figure 1. a) An SEM image of a MoS2 flake on a Si3N4 (20 nm) electron transparent window. b) A Hough image with four highlighted Kikuchi bands. c) A fit of a line profile of one of the highlighted bands in b). The width of Kikuchi line is determined by the distance between the two minima. d) A real-space map of a Kikuchi line's width.

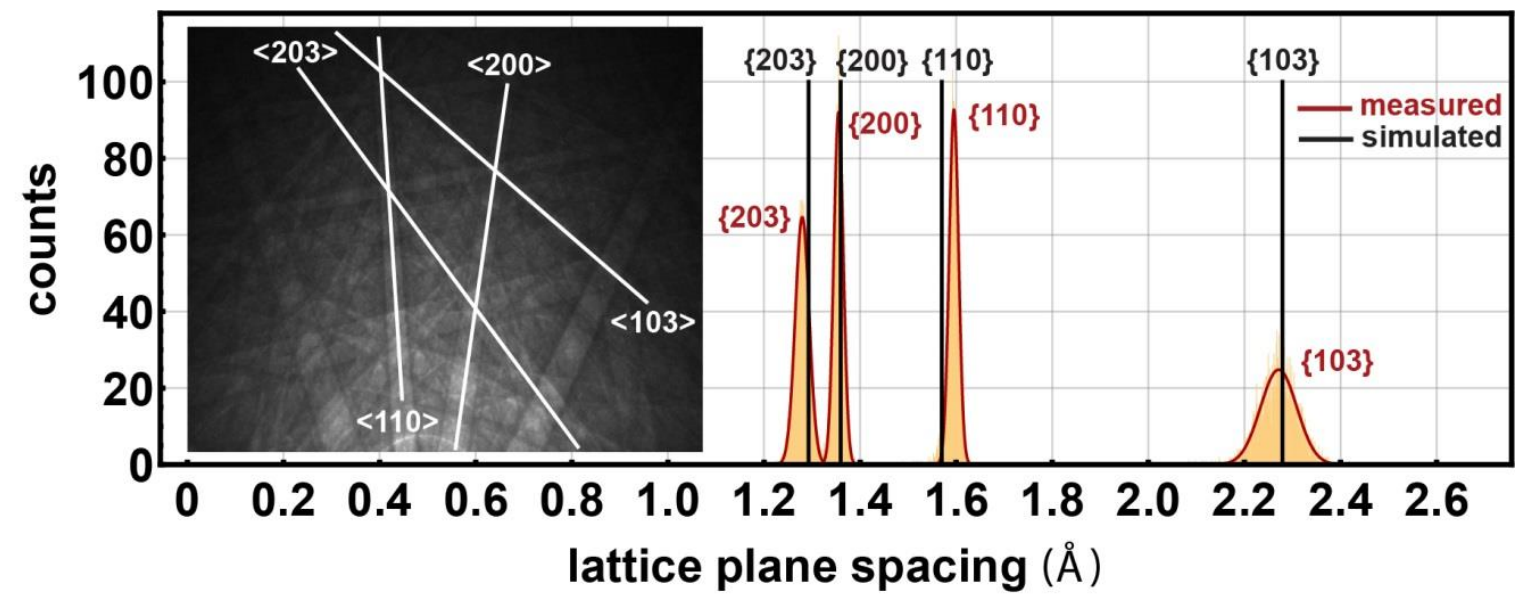

Figure 2. (inset) A Kikuchi diffraction pattern. The four labeled bands were measured using the Hough space image in Fig. 1b. The measured lattice plane spacings are scaled with a coefficient 1.1 and are offset by -0.03 $\AA$ to best match the simulated values. The plotted data is a representative but not complete set of bands.

\section{References}

[1] Majid Abbasi et al., Application of Transmitted Kikuchi Diffraction in Studying Nano-oxide and Ultrafine Metallic Grains, ACS Nano 20159 (11), p. 10991-11002.

[2] Niels Christian Krieger Lassen, Automated Determination of Crystal Orientations from Electron Backscattering Patterns, 1994, p. 51-86.

[3] Hassel O, Zeitschrift fur Kristallographie 61 (1925), p. 92-99.

[4] The data was acquired at the Core Center of Excellence in Nano Image (CNI), University of Southern California. 